\title{
Periodically Poled Silicon (PePSi) for efficient and electronically-tuned nonlinear optics in silicon
}

\author{
Bahram Jalali, Nick K. Hon, Kevin K. Tsia ${ }^{1}$ \\ Electrical Engineering Dept. UCLA, Los Angeles, CA 90095-1594 \\ ${ }^{1}$ Dept. of Electrical and Electronic Engineering, University of Hong Kong, Hong Kong, China \\ jalali@ucla.edu
}

\begin{abstract}
Periodically poled silicon (PePSi) induces substantial $2^{\text {nd }}$ order optical nonlinearity and at the same time achieves quasi-phase matching. PePSi is made by alternating strain gradients along the waveguide using periodic arrangement of stressed cladding layers.
\end{abstract}

OCIS codes: (190.4390) Nonlinear optics: Nonlinear optics, integrated optics; (190.2620) Harmonic generation and mixing.

In addition to its indirect bandgap, silicon's most fundamental optical limitation is its centrosymmetric crystal structure that prevents second-order nonlinearity [1], a requisite property for making electrooptic switches, modulators and parametric wave conversion. Fortunately, perturbation of crystal symmetry gets around the limitation by applying inhomogeneous mechanical stress (strain gradient) to the crystal [2 - 11]. To create efficient nonlinear optical devices,

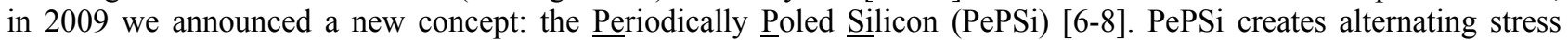
gradients along a silicon waveguide using a periodic arrangement of stressed cladding films (see Figure 1). The structure creates appreciable $\chi^{(2)}$ and simultaneously achieves quasi-phase matching (QPM). Based on experimental $\chi^{(2)}$ values, our simulations have shown efficient MWIR generation $(\sim 5 \mu \mathrm{m})$ through the quasi-phase-matched differencefrequency-generation process (QPM-DFG). The PePSi concept is meant to broaden the capabilities of silicon as a new nonlinear optical medium. This device can also be implemented using silicon nitride or other strained layers, or with piezoelectric material such as PZT on silicon [12-13]. The latter offers the capability to dynamically tune the $\chi^{(2)}$, and the phase matching condition, using on-chip CMOS circuitry. PePSi may also enable THz generation from NIR light through difference frequency generation (DFG).
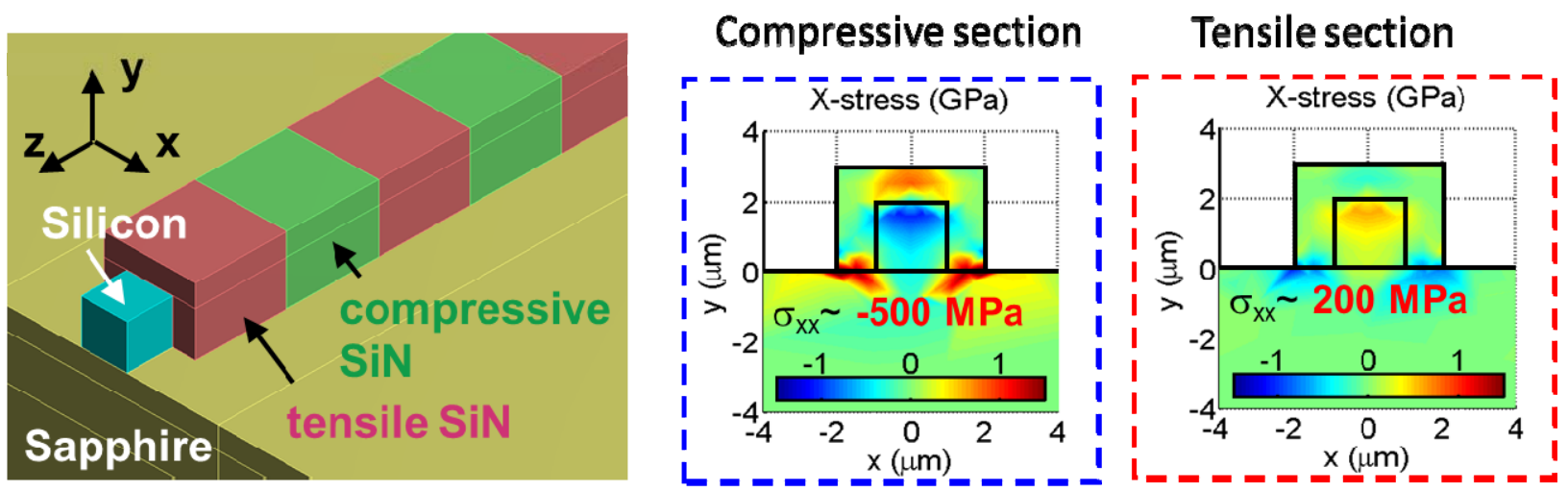

Figure 1 Periodically Poled Silicon (PePSi) for efficient generation of infrared light. PePSi creates alternating stress gradients along a silicon waveguide using a periodic arrangement of stressed cladding films.

Strained silicon has been used to demonstrate linear electro-optic modulators $[4,5,10]$. Based on $\chi^{(2)}$ values reported in $[4,5]$, the stress can be induced by depositing silicon nitride on top of waveguides and a $\chi^{(2)}$ value of $\sim 15 \mathrm{pm} / \mathrm{V}$ has been measured with s GPa stress. PePSi uses strain to not only create $2^{\text {nd }}$ order nonlinearity but also achieve periodic poling (Fig. 1). It creates alternating stress-gradients along a silicon waveguide using a periodic arrangement of anisotropic strained sections created by alternating compressive and tensile films. Fabrication of PePSi uses the fact that the polarity of strain in silicon nitride can be reversed by the deposition conditions and hence a two-step process can be used to create the device. The structure produces appreciable $\chi^{(2)}$ and simultaneously achieves quasi-phase matching (QPM). As an example of the utility of the PePSi technology, we presented our simulation result showing efficient midIR generation through the quasi-phase-matched difference frequency generation process (QPM-DFG) based on a $\chi^{(2)}$ value of $\sim 15 \mathrm{pm} / \mathrm{V}$ [6]. This technology can potentially allow both up- and down- conversion between mid-IR and nearIR for generation and detection using conventional near-IR sources and detectors. PePSi can also enable $\mathrm{THz}$ generation, a new application for silicon photonics that takes advantage of silicon's low loss at THz frequencies. 
Today, there appears to be a difference between the experimental [4,5] and theoretical [3] values of $\chi^{(2)}$ reported in the literature (see Table 1), with theoretical values being much lower than their experimental counterparts. As reported in Ref. 8, we presented a simple intuitive 2D model based on Coulomb's law which shows good agreement with the 3D theoretical work based on bond additivity model [3]. On the other hand, early experimental work [2] reported a measured $\chi^{(2)}$ value on the order of $10 \mathrm{pm} / \mathrm{V}$ which is consistent with later work [4]. The apparent disagreement suggests that there may be important missing physics in the existing theoretical models. At the same time, understanding of silicon's second order nonlinearity is essential for designing nonlinear optical device where the PePSi technology or phase-matched submicron waveguide [11] are needed.

\begin{tabular}{|c|c|}
\hline Theoretical works & Experimental works \\
\hline 2D Coulomb's law model [8] & Jacobson et al. [4] \\
\hline $\begin{array}{c}\left(\chi^{(2)} \text { is } \sim 0.02 \mathrm{pm} / \mathrm{V} \text { under } 1 \mathrm{GPa} \text { stress in the } \mathrm{SiN}\right. \\
\text { strained film) }\end{array}$ & $\begin{array}{c}\left(\chi^{(2)} \text { is } \sim 15 \mathrm{pm} / \mathrm{V} \text { under } 1 \mathrm{GPa} \text { stress in the } \mathrm{SiN}\right. \\
\text { strained film })\end{array}$ \\
\hline 3D bond additivity model [2] & Govorkov et al. [3] \\
\hline $\begin{array}{c}\left(\chi^{(2)} \text { is } \sim 0.03 \mathrm{pm} / \mathrm{V} \text { under } 1 \mathrm{GPa} \text { stress in the } \mathrm{SiN}\right. \\
\text { strained film) }\end{array}$ & $\left(\chi^{(2)}\right.$ is $\sim 10 \mathrm{pm} / \mathrm{V}$ under $1 \mathrm{GPa}$ stress in material $)$ \\
\hline
\end{tabular}

Table 1. Comparison between theoretical and experimental results reported in Ref. 8 (2009)

Recently since 2011, additional experimental measurements of silicon's second order nonlinearity have been reported showing large $\chi^{(2)}$ values. Chmielak et al [10] reported values in the range of 50-120pm/V with an improved electrooptic modulator design. Results show that the values depend on waveguide structure. With optimized submicron waveguide design, the value of $\chi^{(2)}$ can be as high as $100 \mathrm{pm} / \mathrm{V}$ with indications that the nonlinearity maybe higher at the waveguide sidewall. In addition, second-harmonic generation in silicon waveguides has been published recently with $\chi^{(2)}$ values ranging from $0.5-40 \mathrm{pm} / \mathrm{V}$ measured on a micron-sized waveguide with physical model supporting the measurements [9]. Both these recent works suggest experimentally achieved $\chi^{(2)}$ values are even higher than those reported previously.

It is now clear that silicon can be engineered to have $2^{\text {nd }}$ order optical nonlinearity and this will create a new era not just for silicon photonics but for the field of photonics. Despite the discrepancy between experimental and theoretical $\chi^{(2)}$ values, the measured results are consistently large to the tune of at least $10 \mathrm{pm} / \mathrm{V}$ on both submicron and micron sized waveguide. The PePSi ideas shows that strain engineering can not only be used to create but also to perform quasi phase matching. In addition, if the strain is achieved with a piezo-electric thin film, the induced nonlinearity, and the phase matching condition, can be electrically tuned [12-13]. This is particularly useful for actively fine-adjusting the strain in the presence of fabrication errors, temperature or acoustic induced fluctuations.

\section{References}

[1] B. Jalali, "Making Silicon Lase," Scientific American, January 2007.

[2] S. V. Govorkov, et al. "Inhomogeneous deformation of silicon surface layers probed by second-harmonic generation in reflection," J. Opt. Soc. Am. B 6, 1117 (1989).

[3] J. Y. Huang, "Probing Inhomogeneous Lattice Deformation at Interface of $\mathrm{Si}(111) / \mathrm{SiO} 2$ by Optical Second-Harmonic Reflection and Raman Spectroscopy”, Jpn. J. App/ Phys. 333878 (1994).

[4] R. S. Jacobsen, Strained silicon as a new electro-optic material, Nature 441, pp. 199, (2006).

[5] Jacob Fage-Pedersen, Lars H. Frandsen, Andrei V. Lavrinenko, and Peter I. Borel, " A linear electrooptic effect in silicon, induced by use of strain," in Group IV Photonics (IEEE, 2006), pp.37-39.

[6] N. K. Hon, K. K Tsia, D. R. Solli, B. Jalali, “Periodically-Poled Silicon”, APL, 94 (9), 091116 - 091116-3, (2009).

[7] N. K. Hon, K. K. Tsia, D. R. Solli, and B. Jalali, “Addendum: Periodically poled silicon,” APL, Appl. Phys. Lett. 94, 159902 (2009).

[8] N. K. Hon, et al, "Stress-induced $\chi^{(2)}$ in silicon - comparison between theoretical and experimental values" International Conference on Group IV Photonics, San Francisco, CA, (2009).

[9] M. Cazzanelli et al., "Second-harmonic generation in silicon waveguides strained by silicon nitride" Nature Materials (Dec 2011)

[10] B. Chmielak et al. "Pockels effect based fully integrated, strained silicon electro-optic modulator," Optics Express, 19(18), 17212-17219 (2011)

[11] I. Avrutsky et al. "Phase-matched sum frequency generation in strained silicon waveguides using their second-order nonlinear optical susceptibility", Optics Express, Vol. 19, Issue 22, pp. 21707-21716 (2011)

[12] K.K. Tsia, S. Fathpour, and B. Jalali, "Electrical tuning of birefringence in silicon waveguides," Applied Physics Letter, Vol. 92, 061109, pp. 13, February 2008.

[13] K.K. Tsia, S. Fathpour, B. Jalali, "Electrical control of parametric processes in silicon waveguides," Optics Express, Vol. 16 (no.13), pp. 98389843, June 2008. 